\title{
The influence of spicules in the solar radius at multiple radio wavelengths
}

\author{
Caius L. Selhorst ${ }^{1,2}$, Adriana Silva-Válio ${ }^{2}$, Priscila A. Martins ${ }^{2}$, \\ Daiane B. Seriacopi ${ }^{2}$, Pierre Kaufmann ${ }^{2}$ and Hugo Levato ${ }^{3}$ \\ ${ }^{1}$ IP\&D - Universidade do Vale do Paraíba - UNIVAP, Av. Shishima Hifumi, 2911 \\ Urbanova, São José dos Campos, SP 12244-000, Brasil \\ email: caius@univap.br \\ ${ }^{2}$ CRAAM, Universidade Presbiteriana Mackenzie, São Paulo, SP, Brasil \\ ${ }^{3}$ CASLEO, San Juan, Argentina
}

\begin{abstract}
In this work, we analyze observations of the solar radius at 22 and $43 \mathrm{GHz}$ obtained with the $13.7 \mathrm{~m}$ antenna of the Itapetinga Radio Observatory (Atibaia, Brazil) and at submillimeter-wave frequencies, 212 and $405 \mathrm{GHz}$, obtained by the Solar Submillimeter-wave Telescope (SST) (El Leoncito, San Juan, Argentina). The radius is defined as the limb position where the intensity is equal to half of the quiet Sun value. These measured radii are then compared with those predicted by a model of the solar atmosphere proposed by Selhorst, Silva, and Costa (2005). The results show that at 22 and $43 \mathrm{GHz}$, the emission comes from regions high in the chromosphere. Furthermore, the Itapetinga observations yield radii of $985 " \pm 5$ " and $981 " \pm 6 "$ ", at 22 and $43 \mathrm{GHz}$ respectively, consistent with the theoretical positions in the atmosphere. On other hand, the submillimeter observations resulted in a mean radius of 972 " \pm 3 " and $975 " \pm 5 "$ at 212 and $405 \mathrm{GHz}$, respectively, considered equal within the uncertainties. The latter results can be explained by the origin of the emission being very close to the region of minimum temperature, between the photosphere and chromosphere. This is a dynamic region largely affected by many solar features, like spicules and plages.
\end{abstract}

Keywords. Sun: radio radiation, Sun: radius, Sun: spicules

\section{Introduction}

The observations of solar radius at multiple radio wavelengths provide an important tool to study the structure of the solar atmosphere, since different frequencies are formed at distinct atmospheric heights. Moreover the height, with respect to the solar surface, where the emission originates increases with wavelength.

Costa et al. 1999 and Selhorst et al. 2004 showed that the solar radius measured at the chromosphere and layers above it, reflects the solar atmospheric heating during the solar cycle. In the atmospheric model SSC proposed by Selhorst et al. 2005, the authors used the observations of solar radius at $17 \mathrm{GHz}$ to adjust the model, and concluded that the presence of spicules increase the the solar radius at this frequency.

In this work, we observe the solar radius at 4 distinct frequencies $(22,43,212$, and $405 \mathrm{GHz}$ ) studding the solar emission at different atmosphere heights.

\section{Observations}

The observations at 22 and $43 \mathrm{GHz}$ were obtained with the $13.7 \mathrm{~m}$ antenna of the Itapetinga Radio Observatory (Atibaia, Brazil) between 10 and 14 March of 2006, which resulted in 37 maps. 
A total of 92 azimuth maps, obtained by the Solar Submillimeter-wave Telescope (SST) (El Leoncito, Argentina) at 212 and 405 GHz (June - August/2005) were analyzed here.

We defined the solar radius as follow:

- The quiet Sun was take as the most common intensity in the solar disk;

- For each intensity profile the limb was defined as the position where the intensity is half the quiet Sun value taken in two points for each scan;

- These points are then fit by a circumference, which yields a mean radius value.

Table 1. Observational Radius

\begin{tabular}{cc}
\hline $\begin{array}{c}\text { Frequency } \\
(\mathrm{GHz})\end{array}$ & $\begin{array}{c}\text { Radius } \\
(\operatorname{arcsec})\end{array}$ \\
\hline 22 & $985 \pm 6$ \\
43 & $981 \pm 6$ \\
212 & $972 \pm 3$ \\
404 & $975 \pm 5$ \\
\hline
\end{tabular}

The results of mean solar radius are summarized in Table 1. Except for the $405 \mathrm{GHz}$ radius, the observations show that the radius reduces with the increase of the frequency, which agrees with the theory.

\section{Atmospheric Model}

The SSC is a numerical atmospheric model (Selhorst, Silva \& Costa 2005) proposed to reproduce the radio frequencies observations. The authors suggested that an extended chromosphere (up to $3500 \mathrm{~km}$ ) is necessary to reproduce the radio observation, which represents the average effect of small size atmospheric features, such as spicules, for example.

Table 3, summarizes the radius and limb brightening results at the frequencies studied here, after the convolution with a Gaussian beam similar to the antenna resolution. A small limb brightening is predict in the simulations, however, it is not clearly defined by the observations. The simulated radius at 22 and $405 \mathrm{GHz}$ are compatible with the observations, while the radius at 43 and $212 \mathrm{GHz}$ are 1 arcsec outside the measurements even when the error bars are considered.

Table 2. Simulated Radius

\begin{tabular}{cccccc}
\hline $\begin{array}{c}\text { Frequency } \\
(\mathrm{GHz})\end{array}$ & $\begin{array}{c}\text { HPBW } \\
(\operatorname{arcmin})\end{array}$ & $\begin{array}{c}\text { Quiet Sun } \\
(\mathrm{K})\end{array}$ & $\begin{array}{c}\text { Limb Brightening } \\
(\% \text { above the Quiet Sun })\end{array}$ & $\begin{array}{c}\text { Simulated } \\
\text { Radius } \\
(\operatorname{arcsec})\end{array}$ & $\begin{array}{c}\text { Observed } \\
\text { Radius } \\
(\operatorname{arcsec})\end{array}$ \\
\hline 22 & 4 & 9890 & 5 & 983 & $985 \pm 6$ \\
43 & 2 & 8750 & 7 & 974 & $981 \pm 6$ \\
212 & 4 & 6680 & 5 & 976 & $972 \pm 3$ \\
404 & 3 & 5690 & 7 & 976 & $975 \pm 5$ \\
\hline
\end{tabular}

Although the SSC model represents a mean atmosphere, including the influence of atmospheric features, the spicules influence close to the limb differs from those at disc center, since they can reach coronal heights.

The spicules characteristics (density, temperature, height, inclination, and position in the surface) are attributed using the Monte Carlo Method (for more details see Selhorst2005). Figure 1 shows the simulation result for an atmosphere with spicules calculated at $22 \mathrm{GHz}$. The black dots represent the simulation with 1 arcsec resolution, whereas the black curve is the result convolved with a 4' Gaussian beam. 


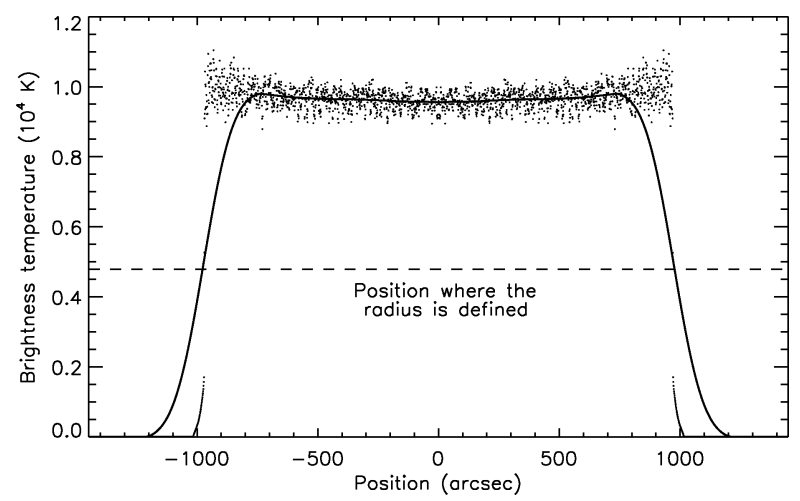

Figure 1. Simulation result for an atmosphere with spicules calculated at $22 \mathrm{GHz}$. The black dots represent the simulation with 1 arcsec resolution, whereas the solid black curve is the result convolved with a 4' Gaussian beam.

In the simulations, we used the spicules physical parameters used in Selhorst et al. 2005 at $17 \mathrm{GHz}$. For these type of spicules the results are:

- reduction of the limb brightening at all frequencies studied;

- increase of the radius at submillimeter waves (212 and $405 \mathrm{GHz}$ );

- the radius at 22 and $43 \mathrm{GHz}$ were smaller than in the simulations without spicules.

Table 3. Simulated Radius with spicules

\begin{tabular}{cccccc}
\hline $\begin{array}{c}\text { Frequency } \\
(\mathrm{GHz})\end{array}$ & $\begin{array}{c}\text { HPBW } \\
(\operatorname{arcmin})\end{array}$ & $\begin{array}{c}\text { Quiet Sun } \\
(\mathrm{K})\end{array}$ & $\begin{array}{c}\text { Limb Brightening } \\
\text { (\% above the Quiet Sun })\end{array}$ & $\begin{array}{c}\text { Simulated } \\
\text { Radius } \\
(\operatorname{arcsec})\end{array}$ & $\begin{array}{c}\text { Observed } \\
\text { Radius } \\
(\operatorname{arcsec})\end{array}$ \\
\hline 22 & 4 & 9600 & 2 & 977 & $985 \pm 6$ \\
43 & 2 & 8690 & 3 & 973 & $981 \pm 6$ \\
212 & 4 & 6740 & 4 & 979 & $972 \pm 3$ \\
404 & 3 & 5790 & 7 & 980 & $975 \pm 5$ \\
\hline
\end{tabular}

\section{Summary}

The results of the simulations can be separated in two parts:

- Model without spicules:

- agrees with the observations of radius at 22 and $405 \mathrm{GHz}$, while at 43 and $212 \mathrm{GHz}$ are 1 arcsec outside of the measured with the error bars;

- presented small limb brightening, between 5 and $7 \%$ above the quiet Sun. These values are within the noise level of the data.

- Spicules influence:

- the spicules reduce the limb brightening in the simulations, except for $405 \mathrm{GHz}$;

- spicules increase the radius at submillimeter frequencies, and reduce the microwave ones. However, the results without spicules are in better agreement with the observations.

\section{References}

Costa, J. E. R., Silva, A. V. R., Makhmutov, V. S., Rolli, E., Kaufmann, P., \& Magun, A. 1999, ApJ, 520, L63

Selhorst, C. L., Silva, A. V. R., \& Costa, J. E. R. 2004, A\&A A, 420, 1117

Selhorst, C. L., Silva, A. V. R., \& Costa, J. E. R. 2005, AESA, 433, 365 